\title{
Heating of adsorbate by vibrational-mode coupling
}

\author{
H. Ueba ${ }^{1}$ and B. N. J. Persson ${ }^{2}$ \\ ${ }^{1}$ Graduate School of Science and Engineering, University of Toyama, Toyama 930-8555, Japan \\ ${ }^{2}$ IFF, FZ-Jülich, 52425 Jülich, Germany
}

(Received 29 August 2007; revised manuscript received 11 October 2007; published 14 January 2008)

\begin{abstract}
We present a theory for the energy transfer between adsorbates and ultrafast laser heated hot electrons in the presence of the vibrational-mode coupling. This mode coupling provides another source of vibrational heating, in addition to the direct heating by hot electrons via friction coupling. The theory is general and accurate and easy to implement to analyze adsorbate motions induced by femtosecond laser heating of the adsorbate and/or metal systems. The heat transfer equation for the coupled harmonic oscillators is applied to the recent timeresolved studies of hopping of carbon monoxide and atomic oxygen on Pt surfaces. It is found that the temperature of the frustrated translation mode responsible for hopping can become high enough, even when its direct friction coupling to the hot electrons is too weak to induce motion.
\end{abstract}

DOI: 10.1103/PhysRevB.77.035413

PACS number(s): 82.53.St, 44.05.+e, 68.43.Pq

\section{INTRODUCTION}

Real-time monitoring of adsorbate motions and chemical reactions induced by femtosecond pulse laser heating of hot electrons in a metal substrate has been a major goal and challenge in surface reaction dynamics. ${ }^{1,2}$ Recently, two different groups carried out real-time studies on lateral motion of adsorbates on Pt, resulting from ultrafast laser-induced heating. Employing time-resolved vibrational spectroscopy to monitor the site-to-site hopping of $\mathrm{CO}$ molecules, Backus et al. ${ }^{3}$ observed the hopping processes, which can be directly related to the vibrational temperatures responsible for hopping. They unexpectedly found that the excitation of the frustrated rotation (FR) mode is essential in the CO hopping process. Excitation of the frustrated translation (FT) mode is significantly too slow to cause the hopping motion of the $\mathrm{CO}$ within the subpicosecond time scale. The reaction pathway involves translational motion, which is already thermally populated in their experiment performed at $100 \mathrm{~K}$. This precursor state couples to the rotational motion excited by ultrafast coupling to hot substrate electrons. The hopping motion is like a dance in which the CO molecules execute concerted rocking and translational steps. ${ }^{4}$

Note that the indispensable role of the FR mode over the FT mode is based on the adsorbate vibrational temperature $T_{a}$ calculated using a heat transfer equation with a friction constant $\eta_{e}$,

$$
\frac{d T_{a}}{d t}=\eta_{e}\left[T_{e}-T_{a}\right],
$$

where the electronic temperature $T_{e}$ is calculated using the well-known two-temperature model. ${ }^{5}$ Stépán et al. ${ }^{6}$ reported that the hopping of atomic oxygen on a Pt surface could not be described using the temperature of the FT mode calculated from Eq. (1). The experimental data were much better reproduced assuming an electron temperature dependence of $\eta_{e}\left(T_{e}\right)$, whose origin has been ascribed to the mode coupling of the O-Pt stretch (excited by the hot electrons) and the translation mode of the $\mathrm{O}$ atom.

These two experimental studies, and the elementary processes suggested therein, motivate us to explore the energy transfer in the presence of the coupling between two different vibrational modes excited by hot electrons. ${ }^{7}$ Such vibrational mode coupling has been demonstrated to play a key role in hopping of $\mathrm{CO}$ on $\mathrm{Pd}(110)$ (Ref. 8) and $\mathrm{NH}_{3}$ on $\mathrm{Cu}(100),{ }^{9}$ where energy transfer from the $\mathrm{C}-\mathrm{O}(\mathrm{N}-\mathrm{H})$ stretch vibration (excited by tunneling electron with scanning tunneling microscope) to the FT mode induces hopping.

Recently, we have presented a theory for the energy transfer between adsorbates and hot electrons using a newly introduced heat transfer coefficient ${ }^{10}$ and pointed out that Eq. (1) is only valid for harmonic oscillators with linear electronvibration coupling (single step-by-step vibrational ladder climbing). Here, we extend that theory and present a general theory of heat transfer between adsorbate and ultrafast laser heated hot electrons in the presence of the vibrational-mode coupling. The population of vibrational levels in the adsorbate system is assumed to be in a local equilibrium so that one can speak about an effective temperature $T_{\nu}(t)$ of the adsorbate vibrational mode $\nu$, which may differ for different adsorbate vibrational modes $\nu$. We are interested in reactions (e.g., desorption or diffusion) involving going over a reaction energy barrier. However, before going over the barrier, an adsorbate will, in general, perform many jumps between its vibrational levels. Thus, the ensemble of adsorbates will be nearly thermalized (except for the levels close to the barrier top where the population is nonequilibrium because of the transfer over the barrier), and we can speak about the (timedependent) adsorbate temperature $T_{\nu}(t)$. We also assume that $k_{B} T_{\nu} \gg \hbar \omega_{0}$ and $k_{B} T_{e} \gg \hbar \omega_{0}$ (where $\hbar \omega_{0}$ is the vibrational quantum of adsorbate mode $\nu$ ). The numerical data presented below indicate that these inequalities are satisfied for the low-frequency modes involved in the adsorbate motions.

\section{THEORY}

We use rate equations to study reaction processes. This is one standard approach frequently used, e.g., it is the basis for Kramer's theory of activated processes and has been used in nearly all studies of reaction induced by hot electrons. The main approximation in this approach is that one assumes fast dephasing processes so one can speak about the occupation 
of the vibrational levels without taking into account the phase of the wave function. The rate coefficients which occur in the rate equation are calculated fully quantum mechanically.

Let $P_{n}$ be the probability of an adsorbate in the vibrational excited state $|n\rangle$. The function $P_{n}(t)$ satisfies the rate equation,

$$
\frac{d P_{n}}{d t}=\sum_{m} P_{m} w_{m \rightarrow n}-\sum_{m} P_{n} w_{n \rightarrow m},
$$

where $w_{m \rightarrow n}$ is the transition rate from the vibrational state $|m\rangle$ to the state $|n\rangle$, caused by the interaction with hot electrons in the metal. We assume local thermal equilibrium on the adsorbate so that

$$
P_{n}=Z_{a}^{-1} e^{-\beta_{a} E_{n}},
$$

where $\beta_{a}=1 / k_{B} T_{a}$ and $E_{n}(n=0,1, \ldots)$. are the vibrational energy levels (for a harmonic oscillator $E_{n}=n \hbar \omega$ ). Substituting Eq. (3) in Eq. (2) and multiplying with $E_{n}$ and summing over $n$ give an evolution of the vibrational energy $Q_{a}$ $=\sum_{n} E_{n} P_{n}$,

$$
\frac{d Q_{a}}{d t}=\sum_{n m}\left[e^{-\beta_{a}\left(E_{m}-E_{n}\right)} w_{m \rightarrow n}-w_{n \rightarrow m}\right] E_{n} P_{n} .
$$

Now, let us consider an adsorbate with two different vibrational modes $\omega_{a}$ and $\omega_{b}$, excited by the hot electrons via the friction coupling $\eta_{a}$ and $\eta_{b}$, respectively. We assume a coupling between the modes so that energy can be directly transferred between the two modes. In order to conserve the total energy, the vibrational transitions are accompanied by the emission or absorption of substrate elementary excitations $\omega_{q}$. We assume the Hamiltonian to be of the form $H$ $=H_{a}+H_{b}+V$, where $H_{a}$ is related to the vibrational mode a (normal mode or reaction coordinate $u_{a}$ ) and similar for $H_{b}$. We also assume that $H_{a}$ commutes with $H_{b}$. The coupling $V$ between modes $\mathbf{a}$ and $\mathbf{b}$ is of the form

$$
V(u)=\sum_{q} \lambda_{q}\left(b_{q}^{+}+b_{q}\right) f\left(u_{a}, u_{b}\right),
$$

where the coupling constant $\lambda_{q}$ is temperature independent. Basically, the nucleus-electron interaction is of the form $\Sigma_{\alpha, \beta} V_{\alpha, \beta}\left(u_{1}, u_{2}, \ldots\right) c_{\alpha}^{+} c_{\beta}$ (where $u_{1}, u_{2}$ are, e.g., normal mode or reaction coordinates of the nucleus and $c_{\alpha}^{+} c_{\beta}$ represents the electronic excitation from level $\epsilon_{\beta}$ to level $\epsilon_{\alpha}$ in the substrate), and since we treat here the electronic excitations as bosons $\left(b_{q}^{+}, b_{q}\right)$, we get terms of the form $\Sigma_{q} V_{q}\left(u_{1}, u_{2}, \ldots\right)$ $\times\left(b_{q}^{+}+b_{q}\right)$. This coupling is also operating in (electronically) cold systems but then the effect we study is negligible. The lifetimes of the low-frequency adsorbate modes are determined by the same coupling, ${ }^{11}$ but with $V_{\alpha, \beta}\left(u_{1}, u_{2}, \ldots\right)$ expanded to linear order in $u_{1}, u_{2}, \ldots$ (this has been studied in great detail in many earlier papers ${ }^{12,13}$ ). The linear order terms, however, cannot give rise to the process of energy transfer between different adsorbate vibrational modes. We therefore consider the leading nonlinear term in the expansion of $V(u)$ which can give rise to mode-mode coupling and energy transfer from one mode to another. The term we study only becomes important when the substrate electron system is very hot.

Let us now calculate the probability rate $w_{n_{a} \rightarrow m_{a}}$ that a vibrational quantum is transferred between modes $\mathbf{a}$ and $\mathbf{b}$, while mode a makes a transition from state $n_{a}$ to $m_{a}$ accompanied with an emission or absorption of a bulk elementary excitation $\omega_{q}$. We get

$$
w_{n_{a} \rightarrow m_{a}}=-\int d \omega\left[\rho_{\lambda}(\omega)-\rho_{\lambda}(-\omega)\right] n(-\omega) W_{n_{a} m_{a}}(\omega),
$$

where $\rho_{\lambda}(\omega)=\Sigma_{q}\left|\lambda_{q}\right|^{2} \delta\left(\omega_{q}-\omega\right)$ is the weighted density of states of the substrate electronic excitations and $n(\omega)$ $=1 /\left(e^{\beta_{e} \omega}-1\right)\left(\beta_{e}=1 / k_{B} T_{e}\right)$ is the Bose-Einstein distribution function, and

$$
\begin{aligned}
W_{n_{a} m_{a}}(\omega)= & \frac{2 \pi}{\hbar} \sum_{n_{b} m_{b}}\left|\left\langle m_{a}, m_{b}\left|f\left(u_{a}, u_{b}\right)\right| n_{a}, n_{b}\right\rangle\right|^{2} \delta\left(E_{m_{a}}+E_{m_{b}}\right. \\
& \left.+\omega-E_{n_{a}}-E_{n_{b}}\right) P\left(n_{b}\right) \\
= & \sum_{n_{b} m_{b}} F_{n_{a} m_{a}}\left(n_{b}, m_{b}, \omega\right) P\left(n_{b}\right) .
\end{aligned}
$$

For a reverse transition, we also obtain

$$
w_{m_{a} \rightarrow n_{a}}=\int d \omega\left[\rho_{\lambda}(\omega)-\rho_{\lambda}(-\omega)\right] n(\omega) W_{m_{a} n_{a}}(-\omega) .
$$

Substituting Eqs. (6) and (8) in Eq. (4) gives a heat transfer equation for the a mode,

$$
\begin{aligned}
\frac{d Q_{a}}{d t}= & \sum_{n_{a} m_{a}} \sum_{n_{b} m_{b}} \int d \omega\left[\rho_{\lambda}(\omega)-\rho_{\lambda}(-\omega)\right] F_{n_{a} m_{a}}\left(n_{b}, m_{b}, \omega\right) \\
& \times\left[e^{-\beta_{a}\left(E_{m_{a}}-E_{n_{a}}\right)} e^{-\beta_{b}\left(E_{m_{b}}-E_{n_{b}}\right)}+\frac{n(-\omega)}{n(\omega)}\right] \\
& \times n(\omega) E_{n_{a}} P\left(n_{a}\right) P\left(n_{b}\right) .
\end{aligned}
$$

We assume electron-hole pair excitations as a substrate heat bath and take $\rho_{\lambda}(\omega)=\bar{\lambda}^{2} \rho^{2}\left(\epsilon_{F}\right) \omega$, where, $\rho\left(\epsilon_{F}\right)$ is the substrate density of states at the Fermi level and $\bar{\lambda}$ some average coupling parameter. Since at high temperatures, $n(\omega) \approx 1 / \beta_{e} \omega$ and $\left[\rho_{\lambda}(\omega)-\rho_{\lambda}(-\omega)\right] n(\omega) \approx 2 \bar{\lambda}^{2} \rho^{2}\left(\epsilon_{F}\right) / \beta_{e}$, we obtain

$$
\begin{aligned}
\frac{d Q_{a}}{d t}= & 2 \bar{\lambda}^{2} \rho^{2}\left(\epsilon_{F}\right) \frac{1}{\beta_{e n_{a} m_{a}}} \sum_{n_{b} m_{b}} \int d \omega F_{n_{a} m_{a}}\left(n_{b}, m_{b}, \omega\right)\left[-\beta_{a}\left(E_{m_{a}}\right.\right. \\
& \left.\left.-E_{n_{a}}\right)-\beta_{b}\left(E_{m_{b}}-E_{n_{b}}\right)-\beta_{e} \omega\right] E_{n_{a}} P\left(n_{a}\right) P\left(n_{b}\right) .
\end{aligned}
$$

Performing the $\omega$ integral gives

$$
\begin{aligned}
\frac{d Q_{a}(t)}{d t}= & \alpha_{a}\left(T_{e}-T_{a}\right)+k_{B} S\left[\left(T_{e}-T_{a}\right) M_{a a} \beta_{a}\right. \\
& \left.+\left(T_{e}-T_{b}\right) M_{a b} \beta_{b}\right],
\end{aligned}
$$

where $S=8 \pi\left[u_{a 0} u_{b 0} \bar{\lambda} \rho\left(\epsilon_{F}\right)\right]^{2}$ ( $u_{a 0}$ and $u_{b 0}$ are the zero point vibration amplitudes of modes $\mathbf{a}$ and $\mathbf{b}$, respectively). Using the analog of Eq. (11) in Ref. 10, we also obtain 


$$
\begin{aligned}
& M_{a a}=\frac{1}{2} \sum_{n_{a} m_{a}} \sum_{n_{b} m_{b}}\left|\left\langle m_{a}, m_{b}|\bar{f}| n_{a}, n_{b}\right\rangle\right|^{2}\left(-E_{m_{a} n_{a}}\right) E_{n_{a}} P\left(n_{a}\right) P\left(n_{b}\right), \\
& M_{a b}=\frac{1}{2} \sum_{n_{a} m_{a}} \sum_{n_{b} m_{b}}\left|\left\langle m_{a}, m_{b}|\bar{f}| n_{a}, n_{b}\right\rangle\right|^{2}\left(-E_{m_{b} n_{b}}\right) E_{n_{a}} P\left(n_{a}\right) P\left(n_{b}\right),
\end{aligned}
$$

where $\bar{f}=f /\left(u_{a 0} u_{b 0}\right)$. In Eq. (11), we have included the contribution of the direct heating in terms of heat transfer coefficient $\alpha_{a}{ }^{10}$

$$
\alpha_{a}\left(T_{a}\right)=k_{B} \eta_{a} \beta_{a} \sum_{n} M_{n_{a}} E_{n_{a}} P\left(n_{a}\right),
$$

where

$$
M_{n_{a}}=\sum_{m_{a}}\left|\left\langle m_{a}\left|\bar{f}\left(u_{a}\right)\right| n_{a}\right\rangle\right|^{2} \frac{\left(E_{m_{a}}-E_{n_{a}}\right)^{2}}{2 E_{n_{a}} \hbar \omega_{a}}
$$

takes into account that when the adsorbate temperature increases, the adsorbate will probe regions in $u_{a}$ far away from the equilibrium position $u_{a}=0$, and this will result in a modified coupling between the adsorbate orbitals and the metal electrons.

In a similar way, we obtain

$$
\begin{aligned}
\frac{d Q_{b}(t)}{d t}= & \alpha_{b}\left(T_{e}-T_{b}\right)+k_{B} S\left[\left(T_{e}-T_{b}\right) M_{b b} \beta_{b}\right. \\
& \left.+\left(T_{e}-T_{a}\right) M_{b a} \beta_{a}\right],
\end{aligned}
$$

where $M_{b a}=M_{a b}$ and

$$
M_{b b}=\frac{1}{2} \sum_{n_{a} m_{a}} \sum_{n_{b} m_{b}}\left|\left\langle m_{a}, m_{b}|\bar{f}| n_{a}, n_{b}\right\rangle\right|^{2}\left(-E_{m_{b} n_{b}}\right) E_{n_{b}} P\left(n_{a}\right) P\left(n_{b}\right) .
$$

Note that $M_{a a}, M_{a b}$, and $M_{b b}$ depend on $T_{a}$ and $T_{b}$, but not on the substrate temperature $T_{e}$. It is also remarked that the second term of Eqs. (11) and (16) does not vanish even for two identical modes $\left(\omega_{a}=\omega_{b}\right)$ with the same temperatures $T_{a}$ $=T_{b}$. This can be understood as follows. Assume first that there are no direct frictional coupling $\left(\eta_{a}=\eta_{b}=0\right)$, i.e., the linear terms in $u_{a}$ and $u_{b}$ in the expansion of $f\left(u_{a}, u_{b}\right)$ in Eq. (5) vanish. Assume now that $T_{a}=T_{b}<T_{e}$. In that case, if the term we derived would vanish when $T_{a}=T_{b}$, then $d Q_{a} / d t$ $=0$, and we would never reach thermal equilibrium in spite of the fact that there is a coupling between the adsorbed molecule and the electronic excitations of the substrate. However, the term we calculated does not vanish unless $T_{e}$ $=T_{a}=T_{b}$, so we will, after long enough time, reach thermal equilibrium as expected because of the coupling to the substrate electrons.

Our derived formulas of heat transfer of Eqs. (11) and (16) are quite general and accurate and easy to implement to analyze adsorbate motions induced by indirect heating via mode coupling, in addition to direct heating via friction coupling to femtosecond laser heated hot electrons. In doing so, however, one may need to know details of electronic struc-
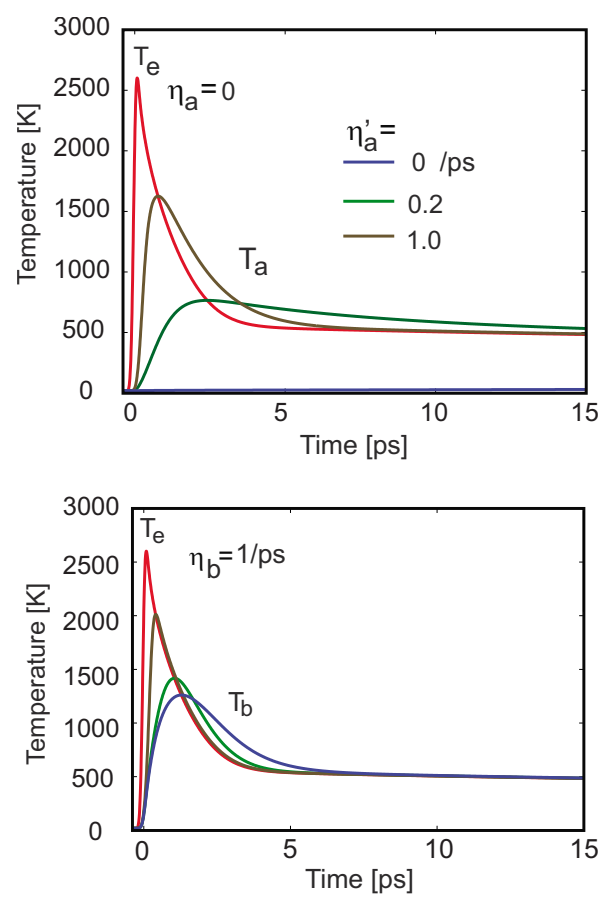

FIG. 1. (Color online) Time dependence of $T_{e}$ (red curve), $T_{a}$, and $T_{b}$. See the text for the parameters used herein.

tures as well as the vibrational wave functions of both modes.

As a simple illustration, assume that both modes can be treated as harmonic oscillators and take the lowest-order coupling $f\left(u_{a}, u_{b}\right)=u_{a} u_{b}$. In this case, we obtain $\alpha_{a}=k_{B} \eta_{a}$ and $\alpha_{b}=k_{B} \eta_{b} \cdot{ }^{10}$ For harmonic oscillator, Eqs. (12), (13), and (17) with $m=n \pm 1$ gives $M_{a a}=\left(\omega_{a} / \omega_{b}\right)\left(\beta_{a} \beta_{b}\right)^{-1}, \quad M_{b b}$ $=\left(\omega_{b} / \omega_{a}\right)\left(\beta_{a} \beta_{b}\right)^{-1}$, and $M_{a b}=\omega_{a} \omega_{b}$, so that we obtain

$$
\frac{d T_{a}(t)}{d t}=\eta_{a}\left(T_{e}-T_{a}\right)+S \omega_{a}\left[\frac{\left(T_{e}-T_{a}\right)}{\omega_{b} \beta_{b}}+\left(T_{e}-T_{b}\right) \omega_{b} \beta_{b}\right]
$$

and a similar equation for $d T_{b}(t) / d t$. We define indirect friction parameters $\eta_{a}^{\prime}=S \omega_{a}$ and $\eta_{b}^{\prime}=S \omega_{b}$ so that $\eta_{a}^{\prime} / \eta_{b}^{\prime}$ $=\omega_{a} / \omega_{b}$. Notice that the second terms in the [.] brackets in Eq. (18) are negligible compared to the first terms (this is confirmed in the numerical calculations shown below). Thus, we can write

$$
\begin{aligned}
& \frac{d T_{a}(t)}{d t} \approx\left[\eta_{a}+\eta_{a}^{\prime} \frac{k_{B} T_{b}}{\hbar \omega_{b}}\right]\left(T_{e}-T_{a}\right), \\
& \frac{d T_{b}(t)}{d t} \approx\left[\eta_{b}+\eta_{b}^{\prime} \frac{k_{B} T_{a}}{\hbar \omega_{a}}\right]\left(T_{e}-T_{b}\right) .
\end{aligned}
$$

Note that the effective friction, $\eta_{a}^{\mathrm{eff}}=\eta_{a}+\eta_{a}^{\prime}\left(k_{B} T_{b} / \hbar \omega_{b}\right)$, depends on the temperature of the coupling partner mode. One expects $\quad S \sim\left(\eta_{a} \eta_{b} / \omega_{a} \omega_{b}\right)^{1 / 2}\left(u_{a 0} u_{b 0} / a^{2}\right)$ so that $\eta_{a}^{\prime}$ $\sim\left(\eta_{a} \eta_{b}\right)^{1 / 2}\left(\omega_{a} / \omega_{b}\right)^{1 / 2}\left(u_{a 0} u_{b 0} / a^{2}\right)$, where $a$ is some atomic distance. Since for low-frequency vibrations $u_{a 0} \sim 0.1 \AA$, we get typically $\eta_{a}^{\prime} / \eta_{a} \sim 0.01\left(\eta_{b} / \eta_{a}\right)^{1 / 2}$. Figure 1 shows the 
time dependence of $T_{a}$ and $T_{b}$ calculated using Eqs. (19) and (20) for $\eta_{a}=0$ (no direct heating of the a mode) ( $\omega_{a}$ $=20 \mathrm{meV})$ and $\eta_{b}=1 / \mathrm{ps}\left(\omega_{b}=50 \mathrm{meV}\right)$ and several strengths of the mode coupling $\eta_{a}^{\prime}=0\left(T_{a}\right.$ remains at $\left.T_{a}=T_{0}\right), 0.2$, and $1 / \mathrm{ps}$, and $\eta_{a}^{\prime} / \eta_{b}^{\prime}=\omega_{a} / \omega_{b}$, where $T_{e}$ (the red curve) is calculated using the two-temperature mode ${ }^{5}$ with a laser pulse width (full width at half maximum) of $0.13 \mathrm{ps}$, a wavelength of $800 \mathrm{~nm}$, and an absorbed laser fluency of $70 \mathrm{~J} / \mathrm{m}^{2}$ irradiated on a Pt metal at the initial temperature $T_{0}=20 \mathrm{~K}$. It is found that $T_{a}$ and $T_{b}$ increase with $\eta_{a}^{\prime}$. The a mode without direct heating can be heated up through the coupling to the $\mathbf{b}$ mode excited by hot electrons. We find that mode coupling can give a strong temperature increase and may give the dominating contribution to the heating of mode a even when its direct coupling $\eta_{a}$ to hot electrons is small, as often is the case, e.g., for parallel adsorbate vibrations.

\section{APPLICATION TO ADSORBATE HOPPING}

As mentioned before, Backus et al. ${ }^{3}$ reported an indispensable role of the FR mode (with an efficient friction coupling to the substrate), rather than the FT mode, in an experiment involving laser-activated hopping of $\mathrm{CO}$ molecules on a stepped Pt surface. This conclusion was based on $T_{a}(t)$ calculated for the FT and FR modes using Eq. (1). Note that in their experiment, the substrate temperature was at $T_{0}$ $=100 \mathrm{~K}$ so that the FT mode is thermally excited, although not to high enough temperature for hopping. We now apply Eqs. (19) and (20) to this system. It should be noted that (in the harmonic approximation) the FT and FR modes of CO on metal surfaces are diagonal modes in a sense that the energy cannot flow from one mode to another. When one includes the coupling to the substrate electronic degrees of freedom, it becomes possible that the energy can flow from one mode to another via substrate electronic excitations. The hopping probability of $\mathrm{CO}$ on a $\mathrm{Pt}(111)$ surface at $T_{0}=100 \mathrm{~K}$ and the laser fluency of $70 \mathrm{~J} / \mathrm{m}^{2}$ is calculated using an Arrheniustype expression $R(t)=k_{0} \exp \left[-U_{0} / k_{B} T_{a}(t)\right]$ for the thermally activated process based on the well-known Kramer's theory. To go over the barrier $U_{0}$, the system must be highly vibrational excited along the reaction coordinate, and in this case, a classical treatment should be a very good approximation. The result shown in Fig. 2 [calculated using the prefactor $k_{0}=1.3 \times 10^{12} \mathrm{~s}^{-1}$, the barrier height $U_{0}=0.4 \mathrm{eV}$, $\omega_{a}(\mathrm{FT}$ mode $)=4.4 \mathrm{meV}, \quad 1 / \eta_{a}=4.0 \mathrm{ps}, \quad \omega_{b}(\mathrm{FR}$ mode $)$ $=51 \mathrm{meV}, 1 / \eta_{b}=0.2 \mathrm{ps},{ }^{3}$ and $\left.1 / \eta_{a}^{\prime}=0.8 \mathrm{ps}\right]$ is in good agreement with the experimental result. Although adsorbatesubstrate energy exchange solely via excitation of the FT mode is significantly too slow to cause the hopping motion of $\mathrm{CO}$, the strong coupling to the FR mode heats up the FT mode high enough for hopping. It is also very important to emphasize that the excitation of the FR mode by hot electrons is absolutely required in order for the FT reaction coordinate mode to get excited within a picosecond for the hopping motion.

Let us now consider femtosecond laser-induced hopping of atomic oxygen on a stepped $\mathrm{Pt}(111),{ }^{6}$ where the anharmonic coupling between the O-Pt stretch mode and the FT mode of an oxygen atom was proposed as a possible origin

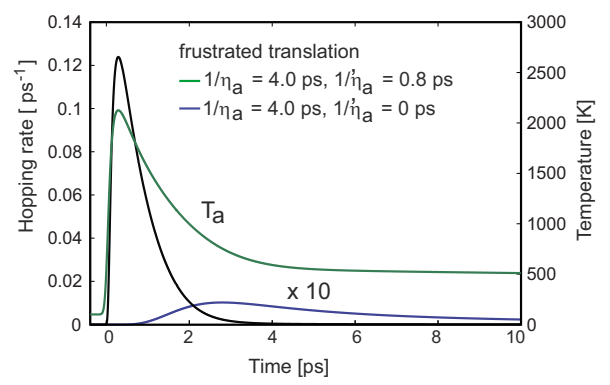

FIG. 2. (Color online) The hopping rate (black curve) and the temperature $T_{a}$ (green curve) calculated for the FT mode $\left(1 / \eta_{b}\right.$ $=0.2 \mathrm{ps})$ with mode coupling $\left(1 / \eta_{a}^{\prime}=0.8 \mathrm{ps}\right)$ to the FR mode $\left(1 / \eta_{a}=4.0 \mathrm{ps}\right)$ and without mode coupling (blue curve, $1 / \eta_{a}^{\prime}$ $=0 \mathrm{ps})$.

of the $T_{e}$-dependent friction coupling in Eq. (1). There is an experimental evidence of this mode coupling in the temperature dependence of the O-Pt stretch mode observed by infrared absorption spectroscopy. ${ }^{14}$ Figure 3 shows an example where modes $\mathbf{a}$ and $\mathbf{b}$ have insufficient friction coupling to the hot electrons. Here, we use $\omega_{a}=50 \mathrm{meV}\left(1 / \eta_{a}=3 \mathrm{ps}\right)$ and $\omega_{b}=60 \mathrm{meV}$, which correspond to the atomic oxygen FT mode and the O-Pt stretch mode on a Pt (111) surface. ${ }^{15} \mathrm{We}$ take $1 / \eta_{b}=1 \mathrm{ps}$ from the low temperature limit of the linewidth of the O-Pt stretch mode. ${ }^{14}$ It is found that $T_{a}$ of the FT mode gets heated high enough with an increase in $\eta_{a}^{\prime}$.

The present analysis suggests quite common elementary processes behind adsorbate motions induced by vibrational excitation with tunneling electrons from scanning tunneling microscopy or hot electrons in the metal substrates. There is a unique vibrational mode having a strong coupling to electrons to receive the energy. Such an accepting mode [the C-O stretch mode and the FR mode for a CO hopping on $\mathrm{Pd}(110)$ and $\mathrm{Pt}(111)$ and the $\mathrm{O}-\mathrm{Pt}$ mode on $\mathrm{Pt}(111)$, respectively] then couples to the promoting mode (FT mode for hopping), since its direct coupling to electrons is too weak to induce the motion.

\section{SUMMARY}

In summary, we have proposed a general formula of heat transfer at surface when there is a coupling between two vibrational modes with different friction couplings to hot

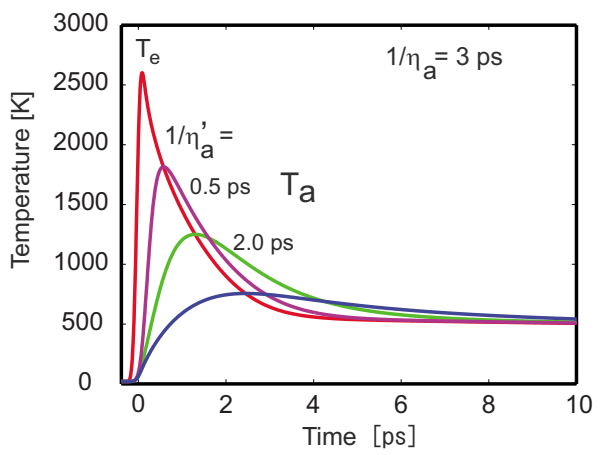

FIG. 3. (Color online) The adsorbate temperature $T_{a}(t)$ without mode coupling (blue curve) and with $1 / \eta_{a}^{\prime}=0.5$ (purple) and $2.0 \mathrm{ps}$ (green) for $1 / \eta_{a}=3 \mathrm{ps}$. 
electrons. Such a mode coupling provides indirect heating or cooling of vibrational modes, in addition to the direct heating due to hot electrons. The theory is general and accurate, and easy to implement to analyze laser femtosecond pulse experiments. The numerical results for harmonic oscillators and a linear coupling to the substrate heat bath (of electron-hole pair excitations) demonstrate a strong heating of the mode even when its direct coupling to the hot electrons is small. Our theory of heat transfer between ultrafast laser heated hot electrons and adsorbates in the presence of the vibrational- mode coupling is a first step toward better understanding of the multidimensional surface reactions of adsorbates on metal surfaces. ${ }^{2}$

\section{ACKNOWLEDGMENTS}

We gratefully acknowledge M. Bonn, U. Höfer, and M. Wolf for many useful discussions and comments. This work is supported by the Grant-in-Aid for Scientific Research (B) from the Japan Society for the Promotion of Science (JSPS).

${ }^{1}$ Laser Spectroscopy and Photo-Chemistry at Metal Surfaces, edited by Hai-Lung Dai and W. Ho (World Scientific, Singapore, 1995).

${ }^{2}$ F. Frischkorn and M. Wolf, Chem. Rev. (Washington, D.C.) 106, 4207 (2006).

${ }^{3}$ E. H. G. Backus, A. Eichler, A. W. Kleyn, and M. Bonn, Science 310, 1790 (2005).

${ }^{4}$ H. Ueba and M. Wolf, Science 310, 1774 (2005).

${ }^{5}$ S. I. Anisimov, B. L. Kapeliovich, and T. L. Perelman, Sov. Phys. JETP 39, 375 (1974).

${ }^{6}$ H. Stépan, J. Güdde, and U. Höfer, Phys. Rev. Lett. 94, 236103 (2005).

${ }^{7}$ H. Ueba, Surf. Sci. 601, 5212 (2007).

${ }^{8}$ T. Komeda, Y. Kim, M. Kawai, B. N. J. Persson, and H. Ueba, Science 295, 2055 (2002).

${ }^{9}$ J. I. Pascual, N. Lorente, Z. Song, H. Conrad, and H.-P. Rust, Nature (London) 423, 525 (2003).

${ }^{10}$ B. N. J. Persson and H. Ueba, Phys. Rev. B 76, 125401 (2007).

${ }^{11}$ B. N. J. Persson and J. W. Gadzuk, Surf. Sci. 410, L779 (1998).

${ }^{12}$ B. N. J. Persson and M. Persson, Solid State Commun. 36, 175 (1980).

${ }^{13}$ M. Head-Gordon and J. Tully, J. Chem. Phys. 96, 3939 (1992).

${ }^{14}$ U. Engström and R. Ryberg, Phys. Rev. Lett. 82, 2741 (1999).

${ }^{15}$ J. Güdde and U. Höfer, J. Phys.: Condens. Matter 18, S1409 (2006). 